\title{
Recovery Oriented Services: Should We Move from Evidence- Based Practice to Value-Based Practice?
}

\author{
Santosh K. Chaturvedi ${ }^{1}$ Jagadisha Thirthalli ${ }^{1}$
}

Published online: 28 July 2015

(C) Springer India Pvt. Ltd. 2015

In the field of mental health rehabilitation, the search for evidence is like chasing an elusive mirage. The community agencies engaging in such work, rarely have opportunities for in-depth monitoring, reflection and learning. They cannot invest in formal research, but need knowledge that is in context, easily accessible, decision oriented, and pragmatic. Thus, they accept much broader range of evidence and share it much more informally. As it is said in the book Paradigms Lost, evidence is in the eye of the beholder. In an applied field, the nature and quality of 'evidence' that is used by programme developers to make programmes can vary widely. (Paradigms Lost: Fighting Stigma and the Lessons Learned by Norman Sartorius, Heather L. Stuart, Julio Arboleda-Flórez).

What can count as evidence in lived experiences is a controversial question. Since most of the work in psychosocial rehabilitation pertains to experiences in living, one comes across mainly experiential evidence. Thus, the debate between evidence based and experience based information raises its head. Moreover, there is currently little evidence that evidence based medicine (EBM) has actually improved patient care. Most clinicians rely more on experience than on research evidence [1].

The process of rehabilitation focuses on increasing competencies or skills and providing environmental supports. In such a situation the focus is on the person as a 'whole' rather than as a 'case'. The rehabilitation plans need to be individualized, both in the process and its outcomes, as, one size does not fit all!

Santosh K. Chaturvedi

skchatur@gmail.com

1 National Institute of Mental Health and Neurosciences (NIMHANS), Bangalore, India
The field of mental health rehabilitation is still evolving and growing. There are limitations of randomized controlled trials (RCTs), especially in the psychosocial intervention arena, that have been well documented. It is difficult to find a uniform sample of persons with comparable mental health problems, as they live different lives. Hence, small sample sizes are available for the complexity of the variables. Further, recovery is multidimensional, making it difficult to measure outcomes.

Research in psychiatric rehabilitation interventions in the developing countries is quite limited. As such, psychiatric rehabilitation services are inadequate except in few apex institutes. There is poor access to psychiatric rehabilitation services. Social stigma towards mental illness is widespread and awareness about mental illness is still poor. In many developing countries, absence of any national insurance system, unemployment benefits or social security drives people away from any available services. Due to this paucity of research, by way of evidence in psychiatric rehabilitation, information is available only for psychoeducation and social skills training. Most studies are hospitalbased, which limit their generalizability. The level of evidence of most of the literature in this field is at a relatively low level. Interventions offered tend to be mainly experienced based rather than evidence based. There is an acute shortage of qualified mental health professionals, especially for rehabilitation. The poor funding from the government towards research projects on psychiatric rehabilitation only makes matters worse.

Implementing evidence based practices (EBPs) in the field of rehabilitation can be a challenge. In order to create a demand for EBPs, one would need to raise awareness of the potential benefits and utility of EBPs. This would have to be directed at all stakeholders including clients, family members, administrators, practitioners, clinicians, 
supervisors and researchers, and could be accomplished by lectures, conferences, journal articles, academic and training centers. One may question whether it is possible to select most appropriate EBPs and prepare for the delivery of a new EBP at the level of the organization and of the practitioner.

Criticisms of evidence-based practices for rehabilitation in psychiatric practice need to be acknowledged. There are complex issues concerning the methods and criteria for identifying EBPs. Differentiating between "evidencebased" and "emerging" practices centers primarily on the rigor of the research designs. There are differing levels of empirical support for various EBPs. Some EBPs have been characterized as paternalistic and coercive and as mentioned above, there is little evidence that EBM has actually improved patient care! In evidence-based practices important philosophical elements of Recovery oriented services may be omitted. The elements of recovery oriented services may not be empirically linked to the traditional outcomes reported. Such services can significantly alter the consumer's personal experience of the program and thus his/her unique process of recovery. A large part of the current practice in psychosocial rehabilitation is based on the type of evidence considered lower in the hierarchy of EBM, this is particularly so for psychological and psychosocial therapies. On the other hand, there are treatment methods with proven efficacy, which do not seem to have reached clinical practice. The generalizability of evidence is a limitation. Incorporating patient preferences and shared decision-making and informed choices often becomes difficult in EBPs. Inadequate access and utilization of information technology, together with aggressive marketing and promotion by the pharmaceutical industry (international and national), make the perception of EBM in psychosocial interventions to be a distant dream.

The concept of value based practices has emerged recently in Medicine. Value based practices promote combination of EBPs and recovery oriented services in a manner which are perceived to be meaningful to the patient. EBPs guided by the values of recovery oriented services can be powerful tool for helping consumers pursue personally important recovery goals. Clients should be able to make informed decisions about which services including EBPs they want to use in their own recovery plan. Combination of all the above interventions guided by principles of recovery oriented services is the best value based practice. Audit based practice may make more sense than evidence based practice for rehabilitation.

The current, and the third issue of the Journal, has a variety of articles, which can add to the evidence and value for psychiatric rehabilitation. This issue has two remarkable guest editorials, one on modulating neuroplasticity with brain stimulation for cognitive rehabilitation in persons with schizophrenia, and another on psychiatric needs of families in which one or both parents suffers from mental illness. The latter editorial was 'prompted' by two articles on individualism and collectivism, published in the Journal. Other articles include one on the effect of trauma from an armed conflict on disadvantaged children in Philippines, and the role of psychosocial workers in responding to such children's psychosocial needs. Another on an exploratory evaluation of co-produced rehabilitative social groups in English community psychiatric services, from the UK, which showed that co-production improved self-esteem, empowerment and purpose, and enabled recovery for service user co-producers. There is a paper from USA on two case studies that examine how participatory public art promotes recovery and wellness. A study from Nigeria, presents the profile of functional impairment and the influence of psychopathology among individuals with schizophrenia in a Nigerian tertiary health facility. Another study from USA identifies factors that positively and negatively impact the referral to and utilization of peer support services by traditional providers and strategies for achieving optimal utilization. A report from Bangalore presents an understanding of the nature and pattern of employment among persons with severe mental disorders and compared those who sustained versus lost the job after onset of illness. Another one is on the negative impact of stigma on employability of persons with schizophrenia from Kolkata, and how intervention proved helpful. Understanding emotions in a person with intellectual handicap and how to provide rehabilitation counselling in a single session are other contributions. There is also a user perspective account, which is very informative. It is heartening to note the contributions from so many countries across the world in this issue, which indicates that authors find this journal as an avenue to publish their important observations, and add to evidence base as well as experience base.

Santosh K Chaturvedi

Jagadisha Thirthalli

Editors-in- Chief

\section{Reference}

1. Fava GA, Guidi J, Rafanelli C, Sonino N. The clinical inadequacy of evidence based medicine and the need for conceptual framework based on clinical judgement. Psychother Psychosom. 2015;84:1-3. 